\title{
Prognostic value of the neutrophil-to-lymphocyte ratio and primary tumor location in patients with EGFR-mutated metastatic non-small cell lung cancer treated with TKIsNote: We found that we got a wrong results in the previous article. So, we checked our collected database and calculated again and corrected now.
}

\section{Xueqi Xie}

Shandong First Medical University

Xiaolin Li

Shandong Cancer Hospital and Institute

Wenjie Tang

University of Jinnan Shandong Academy of Medical Sciences

Jinlong Chen

Shandong Cancer Hsptal and Institute

Minghuan Li

Shandong Cancer Hospital and Institute

Peng Xie ( $\nabla$ xiepengro@126.com )

Shandong Tumor Hospital and Institute https://orcid.org/0000-0001-8647-2882

Jinming Yu

Shandong Cancer Hospital and Institute

Research article

Keywords: Epidermal growth factor, NSCLC, TKI, Primary tumor location, NLR, PFS

Posted Date: December 16th, 2020

DOl: https://doi.org/10.21203/rs.3.rs-29351/v4

License: (a) (i) This work is licensed under a Creative Commons Attribution 4.0 International License. Read Full License 


\section{Abstract}

Background: Targeted therapy with the epidermal growth factor receptor (EGFR) tyrosine kinase inhibitors (TKIs) has improved the field of metastatic non-small cell lung cancer treatment. Higher neutrophil-tolymphocyte ratio (NLR) and lower relative lymphocyte counts as inflammatory indicators and associated with worse overall survival and progression free survival (PFS) in several tumor types. Few studies focused on these inflammation markers in field of TKIs eras.

Methods: Patients with advanced EGFR mutation NSCLC treated with TKIs were included. Pre-treatment NLR means neutrophil to lymphocyte ratio measured in peripheral blood within one week before treating with TKIs. The baseline clinical characteristics of each group were compared by chi-square and $t$ tests. Cox regression analyses were used to evaluate prognostic value of peripheral blood parameters on progression free survival (PFS). All prognostic factors were explored with multivariable regression.

Results: We retrospectively analyzed 221 patients with metastatic NSCLC harboring exon 19 deletion, 21 L858R or rare mutation and receiving TKls. Finally, a total of 190 patients were analyzed. The optimal cutoff values for pretreatment absolute lymphocyte count (Lym), lymphocyte percentage (Lym\%), absolute neutrophil count (Neu), the percentage of neutrophil granulocytes (Neu\%) and NLR were 1.625, $18.8 \%, 3.675,51.8 \%$ and 4.965 , respectively. Patients with high neutrophil percent (18.8 months vs 13.0 months, $\mathrm{P}=0.003)$, absolute neutrophil counts ( 12.0 months vs 14.5 months, $\mathrm{P}=0.014)$ and NLR (7.0 months vs 15.2 months, P<0.001, 1-year PFS Rate, $38.4 \%$, respectively) had worse PFS. In contrast, patients with high absolute lymphocyte counts ( 16.5 months vs 13.0 months, $P=0.012$ ) and lymphocyte percent (15.3 months vs 8.8 months, $P<0.001)$ had a better progression-free-survival. Besides, tumor location is also an important factor for prognosis ( 14.3 months vs 11.6 months, $P=0.003$ ). On multivariate analysis, NLR and primary tumor location were both identified as independent and significantly risk indicators for worse PFS.

Conclusion: NLR and primary location are both independent prognostic factors for PFS in patients with metastatic EGFR mutated lung tumor. Whether or not NLR and primary location could be useful markers in efficacy prediction of TKIs in advanced NSCLC calls for further investigation. KEYWORDS: Epidermal growth factor; NSCLC; TKl; Primary location; NLR; PFS

\section{Background}

Lung cancer continues to be the leading cause of cancer-related deaths worldwide, especially in patients with metastatic disease [1]. Non-small cell lung cancer (NSCLC) as the most common type of lung carcinoma, accounts for approximately $85 \%-90 \%$ of all lung cancer cases. Lung neoplasms can also be grouped into the central and peripheral subtypes according to primary tumor location [2]. Pulmonary adenocarcinoma pathological subtypes have replaced squamous cell carcinoma in recent years, often located in the periphery, as one of the most common cell types of lung cancer. In recent years, a number 
of previous studies have shown that primary tumor location is an important factor to guide treatment schedules and predict clinical prognosis in lung tumors[3-6].

Advances in epidermal growth factor receptor tyrosine kinase inhibitors EGFR-TKIs of non-small cell lung cancer (NSCLC) have led to a new era of target therapy, particularly in patients with NSCLC who have EGFR mutations. Until recently, targeted therapy has remained the first-line treatment for the majority of patients with targetable oncogenic driver alterations. In recent years, gefitinib, erlotinib, afatinib, and osimertinib have shown better clinical outcomes and response rates than chemotherapy using cytotoxic drugs.

Host immunity may affect prognosis in patients with various cancers. Evidence has demonstrated that immune system plays an important role in promoting anti-tumor defense. Tumor-associated inflammation and tumor microenvironment play a critical role in cancer development, progression and metastasis[7-9]. The neutrophil-to-lymphocyte ratio (NLR) is one of the most widely used inflammation biomarkers in solid cancers and can be easily calculated from routine blood examination results. Inflammation not only can contribute to development of various cancers, but also is recognized as a hallmark of cancer[10]. For example, several peripheral blood parameters, including markers of systemic inflammation such as baseline the NLR, the lymphocyte-to-monocyte ratio (LMR) and absolute lymphocyte count (Lym) have been associated with survival in patients with metastatic lung neoplasms treated with the ICls[11, 12].

Neutrophil counts, lymphocyte counts and NLR play significant roles in the inflammatory response and demonstrates their value in various of solid tumors. As people costs much in treatments, inexpensive, readily available and more effective potential prognostic markers need to be used to assist the prognosis and risk stratification of patients with the lung cancer. Previous studies have shown that the NLR can be considered as a predictor to evaluate the prognosis, which can be used in the EGFR-TKIs and ICls [13-15]. Few studies have found and association between NLR and OS. There is a lack of understanding of the relationship between peripheral blood counts and progression-free survival (PFS), particular in patients treated with TKIs.

The aim of our study was to find the prognostic value of pre-treatment complete blood count (CBC) parameters in NSCLC patients with advanced NSCLC treated with EGFR-TKIs (as first-line to third-line therapy). Additionally, we needed to find various determined factors to predict clinical outcomes. In general, the main objective was to explore more effective, useful and noninvasive predictors to assess the benefits of patients receiving TKI treatment.

\section{Methods}

\subsection{Patients and clinical characteristics}

This retrospective study was approved by review board of Shandong Cancer Hospital and Institute. We respectively analyzed patients with metastatic or recurrent postoperative NSCLC. All patients needed to 
meet the following standard criteria: 1) were of 18 years or older, 2) pathologically proven adenocarcinoma with a positive EGFR mutation test before starting any treatment, including chemotherapy, surgery, radiotherapy (RT) and targeted therapy, 3) complete medical records/computed tomography (CT) scans of the chest and/or positron emission tomography (PET) scans/bronchoscopies, 4) treatment with TKI drugs as the first-, second- or third-line therapy, 5) results of complete peripheral blood test, including neutrophil and lymphocyte counts, within 1 week before receiving EGFR-TKI treatment and 6) received TKI drugs including Gefitinib, Erlotinib and Icotinib. Thus, patients who meet all the above-mentioned criteria were included from the electronic record system.

The clinical stage was determined by the 7th edition of the American Joint Committee on Cancer (AJCC)/Union for International Cancer Control (UICC) staging system. Pre-treatment NLR means the nearest peripheral absolute neutrophil and absolute lymphocyte ratio before starting of TKIs within 1 week. PFS was defined as the time from treatment with the EGFR-TKIs to the terminology or pathology evidence of progression or recurrence. OS was measured from the day of NSCLC diagnosis to the date of death from any.

\subsection{EGFR mutation test}

A total of 190 specimens before receiving EGFR-TKIs were obtained via tissue biopsy, including bronchoscopy, CT-guided biopsy, or surgical procedures from primary or metastatic sites. If we could not obtain the tissues, we collected peripheral blood and metastatic body fluid. The majority of samples use the peptide nucleic acid (PNA)-mediated polymerase chain reaction (PCR) clamping method. Few samples were sequenced with targeted next-generation sequencing (NGS) of 18 lung cancer gene panels.

\subsection{Tumor location evaluation}

According to results of patients' CT imaging, bronchoscopy, or both, we identified tumor as central and peripheral lung cancer. As there is no standard definition to classify peripheral and central lung tumor. We defined central tumors as occurring from segmental or proximal bronchi. As for peripheral type, we considered that tumors in subsegmental or other distal bronchi and bronchioli according to previous studies $^{12}$. All images were anonymized and blindly evaluated by one radiologist and one oncologist. For discordant cases, a second oncologist would evaluate the images.

\section{Statistical Analyses}

Pre-treatment blood data were obtained from electronic medical records. Student's t-test was used to evaluate the difference in absolute lymphocyte counts, relative lymphocyte counts and relative neutrophil counts in two different groups. We used Mann-Whitney $U$ test for the ordinal data and Pearson's $\chi 2$ or Fisher's exact test to compare clinical characteristics at baseline. EGFR mutation status and primary tumor location (central vs. peripheral) were analyzed using Pearson's chi-square tests. Receiver operating characteristic (ROC) curve analysis was used to determine the optimal value of Lym, percentage of lymphocytes (Lym\%), absolute neutrophil count (Neu), the percentage of neutrophil granulocytes (Neu\%) 
and the NLR in terms of their association with PFS. Patients and clinical characteristics were summarized with descriptive statistics. We also used the Kaplan-Meier method and log-rank for univariate survival analysis. Multivariate Cox regression analysis was used to analyze the effect of different clinical characteristics on PFS and OS, and statistical analyses were performed with SPSS 19.0. P<0.05 was considered statistically significant.

\section{Results}

\subsection{Patients and tumor characteristics}

From January 2014 to November 2018, a total of 221 patients were treated with TKIs. Thirty-one patients did not meet the eligible requirements (complete peripheral blood tests were missing for 29 patients, the primary tumour location was difficult to define in the imaging data for 1 patient, and imaging data before starting TKIs were missing for 1 patient). Baseline characteristics of the patients are summarized in Table 1. The majority of patients were female $(\mathrm{N}=122)$, and the median age was 58.0 years (range: 30 - 87 years), a total of 148 patients had never smoked. A total of 172 patients underwent PCR tests and 18 underwent NGS tests. We also found the most oncogenic alteration was EGFR L858R mutation ( $N=110)$. Additionally, 83 patients had bone metastasis (43.7\%), 26 (13.7\%) had liver metastasis, and 59 (31.1\%) had brain metastasis. In addition, many patients received chemotherapy as the first-line therapy $(\mathrm{N}=90)$. 27 patients underwent surgery before targeted therapies. No correlations were found between primary location and EGFR mutation status. (Pearson's chi-square= 0.76, $\mathrm{P}=0.963)$ ) 142 patients had peripheraltype adenocarcinoma. There were 164 patients with stage IV lung cancer. A total of 26 patients had postoperative recurrence.

\subsection{Optimal cutoff value for CBC}

Median PFS was 12.51 months when we used PFS as an end point for blood routine. According to ROC analysis, we obtained the following optimal cut-off values: 1.625 for pre-treatment Lym, $18.8 \%$ for pretreatment Lym\%, 3.675 for pre-treatment Neu, 51.8\% for pre-treatment Neu\% and 4.965 for pre-treatment NLR for PFS (Table 3). Among the many routine peripheral blood indicators, pre-treatment Lym had the largest AUC, which was 63.2\%. However, the pre-treatment Neu\% had the lowest AUC, which was 49.9\%, indicating its low predictive value.

\subsection{Association between pre-treatment CBC and clinicopathological factors}

As Table 4 demonstrates, a positive association between the dichotomized NLR and Neu was found, with a kappa value of $0.487(P<0.0001)$. Additionally, in Table 4, a negative correlation between the dichotomized NLR and Lym were consistently explored, with a kappa value of $0.310(P<0.0001)$. In addition, we also found that there were positive correlations between bone metastasis and pre-treatment Neu\% and NLR ( $P=0.007$ and $P=0.022$, respectively). There were negative correlations between pretreatment Lym\% and bone metastasis $(P=0.029)$. The absolute pre-treatment Lym tended to correlate with 
PFS $(P=0.007)$. Additionally, there were no correlations between other blood biomarkers and metastasis sites. We did not find a relationship between peripheral blood biomarkers and primary tumour location.

\subsection{Prognostic factors for PFS}

The median PFS was significantly related to the pre-treatment Lym, Lym\%, Neu, Neu\% and NLR. In summary, the PFS of patients with a higher Lym (HLym) and higher relative lymphocyte count HLym\% was significantly higher than those of the patients with LLym and LLym\% $(P<0.05 ;$ Fig. $1 A, B)$. In contrast, patients with $\mathrm{HNeu}, \mathrm{HNeu} \%$ and HNLR had poorer PFS than those with LNeu, LNeu\% and LNLR $(P<0.05$; Figs. 1C, 1D; and 2). We also found that peripheral-type lung neoplasms had a better clinical outcome than central-type lung neoplasms $(P=0.003$, hazard ratio $(H R)=1.739,95 \%$ confidence interval $(C l)=$ 1.207-2.506) (Fig. 2). Pre-treatment blood tests were found to be important for PFS, patients with Lym $<1.625$ had shorter PFS than those with Lym $\geq 1.625$ (13.0 months and 16.5 months, $P=0.012$, $\mathrm{HR}=0.662,95 \% \mathrm{Cl}=0.480-0.915)$. Median PFS was 8.8 months in patients with $\mathrm{Lym} \%<18.8 \%$, while the PFS was 15.3 months in patients with

Lym\% $\geq 18.8 \%$. (P凶0.001, $\mathrm{HR}=0.391,95 \% \mathrm{Cl}=0.274-0.560)$ Besides, patients with a $\mathrm{HNeu}$, relative neutrophil count and HNLR had a worse clinical benefit $(P=0.015$, HR 1.481, 95\% $\mathrm{Cl} 1.081-2.030 ; P=0.003, H R$ 1.824, HR 1.223-2.720; $P \llbracket 0.001, \mathrm{HR} 4.996,95 \% \mathrm{Cl} 3.189-7.826)$. We also did not find the association between EGFR mutation and PFS ( $P=0.368)$. EGFR mutations mainly consist of the exon 19 deletion (del19) and the L858R point mutation. The other mutations are not as common.

We used multivariate Cox regression analysis to evaluate the independent prognostic predictors. Cox regression analysis demonstrated that NLR and, primary tumor location were related to PFS and can be considered as independent factors for predicting poor prognosis $(P=0.001, H R=3.297,95 \% C l=1.614$ 6.737; Pख0.001, HR=2.021, 95\%Cl=1.365-2.993; respectively) (Fig.3).

\section{Discussion}

Systemic inflammation plays a critical role in tumor proliferation and metastasis. Our study demonstrated that pre-treatment NLR ( $\geq 4.965)$ and primary tumor location were independently and significantly associated with the shorter PFS in patients with EGFR-mutated metastatic NSCLC. In univariate analyses, NLR, Lym, Lym\%, Neu, and Neu\% all played an important role. In addition to the NLR, previously determined related and effective prognostic factors include tumor size, sex, disease status, tumour location and performance status [2]. As the NLR as an inflammatory marker plays an increasingly role, it is widely analyzed in the solid tumors, such as ovarian cancer, urothelial carcinoma, head and neck cancer, lung cancer, hepatocellular carcinoma and so on[16]. The majority of the analyses focus on the multiple comprehensive treatments, including chemotherapy, chemoradiotherapy, radiation therapy, surgery, immunotherapy and immunotherapy combined with radiotherapy[13, 17-22]. The mechanism reflects the patients' inflammatory and systemic immune status. However, in a subset of EGFR-mutant advanced disease, NLR was an important factor to assess the prognosis when treated with chemotherapy as first-line therapy. There are few studies on patients treated with targeted therapies, 
particularly EGFR-TKIs. Only a few studies have shown that the NLR is a significant prognostic factor for PFS in patients who receive TKIs. Our study complements the current studies in this field $[15,23]$.

To the best of our knowledge, inflammation can be regarded as the hallmark of cancer, and plays an integral role in tumorigenesis, lymphomagenesis and progression of cancer. Increasing evidence showed that elevated inflammation was related to poor cancer-specific in a variety of tumors[16, 24]. Tumor cells can lead to upregulation of the inflammatory process, which can release the proinflammatory factors, promoting the cancer cell proliferation, angiogenesis and lymphagionesis. The inflammatory cells and factors, including the lymphocytes, neutrophils, platelet, IL-6, IL-8 and C-reactive protein (CRP) have different influences in various cancers [25-27]. Neutrophils and macrophages can secret tumor growth factors, like the TL-4, IL-8 and vascular endothelial growth factor (VEGF), which can stimulate the tumor microenvironment. Yosuke Morizawa et al analyzed the correlation of tumor microenvironment and NLR in blood samples with muscle bladder cancer, particularly in immune cells and cytokines. They found that preoperative NLR was associated with immunohischemical expression of forkhead box P3 (Foxp3) in bladder cancer.In addition, they also suggest that IL- 6 and IL-8 produced by cancer cells influence the level of NLR in patients with bladder cancer[26]. The same conclusion can also be found in head and neck squamous cell carcinoma, Ming-Shao Tsai et al demonstrated that NLR was positively related to the expression levels of IL-6 and PD-L1 expression[27]. Lymphocyte, especially the tumor-infiltrating lymphocytes (TILS) have a significant effect not only on the lung cancer but also on other solid tumors. The relationship between peripheral blood counts and CD8+ TILs has been found in breast cancer[28]

In NSCLC, TILs play a significant role in the response to anti- PD-1 therapy in patients with metastatic. The more activated CD8+ cells there are, the better the tumor can be controlled by cytotoxic activity and by inducing apoptosis of cancer cells [29]. Lymphocyte counts are also used to assess the prognosis in

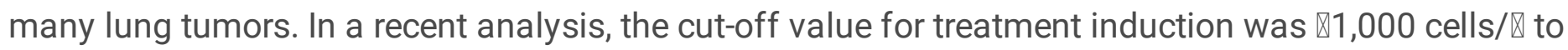
evaluate the clinical benefits treated with immunotherapy combining (RT) [30]. Preoperative lymphocyte counts are considered to be favorable prognostic factors in NSCLC to predict the disease-free survival[29]. In our study, we found that Lym\% play a significant role in the PFS. The higher the elevated relative lymphocyte counts are, the better the clinical benefits are in patients receiving first-, second and third-line TKIs.

In the inflammatory response to cancer, neutrophils may play a role as reservoirs for circulating vascular endothelial growth factor and promote metastasis. Previous studies have shown that circulating neutrophils release various inflammatory factors, including tumor necrosis factor- $a$ (TNF- $a$ ) and IL-6, to promote tumor progression [7]. In our study, we also found that the higher absolute neutrophil counts and relative neutrophil counts were associated with shorter PFS.

Leukocytes include lymphocytes, monocytes, neutrophils, eosinophils and basophils. NLR would be a simple, inexpensive and reliable pre-treatment prognostic factor for patients treated with TKIs. Iseki et al showed that Lym\% was affected by neutrophils and monocytes, which is the reason why Lym\% reflects systemic inflammation more accurately than Lym[31]. The results are consistent with our conclusions 
from univariate analysis. Previous analyses shows that NLR can be used as an independent prognostic factor when patients receive gefitinib or erlotinib as a first-line or second-line therapy [22]. Multivariate Cox regression analyses showed that a higher pre-treatment NLR was associated with worse PFS. Besides, univariate analysis demonstrated that a LNLR at baseline was associated with a better prognosis in EGFR-mutated metastatic NSCLC. Further prospective investigations with adequate samples are needed to fully understand the prognostic value of the pre-treatment NLR.

The results from our retrospective study supports those form previous studies, showing that the NLR is a significant factor for prognosis of NSCLC. Additionally, our reports are the first to demonstrate that NLR and primary location can both be regarded as important prognostic factors in EGFR-mutated advanced NSCLC as first-, second-, and third-line therapies. An increasing number of findings have shown that primary tumor location is one of the determining factors for choosing the optimal treatment for and estimating the prognosis of patients with an advanced tumor. In our study, we used definitions according to previous findings in CT scans and bronchoscopies. A high portion of patients with peripheral adenocarcinoma show clinical benefit compared to patients with central adenocarcinoma. Wang et al has investigated that central adenocarcinoma has a worse prognosis compared with central adenocarcinoma, which consistent with our conclusions[6]. EGFR mutation status can also be considered as a prognostic factor for treatment of TKIs as a first-line treatment in advanced NSCLC. According to a previous analysis, patients with an EGFR del19 had longer PFS than those with EGFR exon 21 mutation. Jiang et al concluded that EGFR mutation status is a good predictor for patients treated with EGFR-TKIs in NSCLC[32]. A large meta-analysis on patients who were treated with first-line TKIs also revealed that patients with an EGFR exon 21 mutation had a shorter PFS than patients with an EGFR del19[33]. However, in our research, we compared three different EGFR statuses and did not have the same conclusions. We concluded that the NLR and primary tumor locations are both predictive factors for the efficacy of EGFR-TKIs as first-, second- and third-line therapies.

We are aware that there are some limitations in our analysis. First, as a retrospective study, we have some selection biases. Although patients' data concerning the laboratory, CT scans/PET-CT and survival data were complete, there was also a patients' selection bias. Second, the relative numbers of eligible patients were small. In summary, the Lym, LLym\%, HNeu, HNeu\% and higher HNLR, corelated with poorer prognosis of NSCLC patients treated with TKIs. The NLR and peripheral-type tumor seem clinically meaningful for patients treated with EGFR-TKIs. As an effective and prognostic biomarker, NLR is inexpensive and readily available. We need further investigations with a large prospective study to validate our results in the future.

\section{Conclusion}

The NLR and primary tumor location are both effective and meaningful factors for EGFR-mutated advanced lung cancer (Fig3). The primary tumor location is also a significant predictor for treatment planning. We also found that NLR was a useful predictor for systemic inflammation in patients treated with TKIs. However, we need more data to explore understand the relationship among these parameters. 
Our findings support the existing hypothesis that systemic inflammation is associated with clinical outcomes.

\section{Abbreviations}

EGFR: epidermal growth factor receptor; TKIs: tyrosine kinase inhibitors; NLR: neutrophil-to-lymphocyte ratio; PFS: progression free survival; NSCLC: non-small cell lung cancer; Lym: absolute lymphocyte count; Lym\%: percentage of lymphocytes; Neu: absolute neutrophil count; Neu\%: the percentage of neutrophil granulocytes; LMR: lymphocyte-to-monocyte ratio; ICls: immune checkpoint inhibitors; OS: overall survival; CBC: complete blood count; CT: Computed tomography; PET: position emission tomography; PNA: peptide nucleic acid; PCR: Polymerase Chain Reaction; NGS: next generation sequencing; ROC: receiver operating characteristic; AUC: Area Under the Curve; HLym: higher in absolute lymphocyte counts; HLym\%: higher in relative lymphocyte counts; LLym: lower in absolute lymphocyte counts; LLym\%: lower in relative lymphocyte counts; $\mathrm{HNeu}$ : higher in absolute neutrophil count; $\mathrm{HNeu} \%$ : higher in the percentage of neutrophil granulocytes; HNLR: higher in NLR; LNEU: lower in absolute neutrophil count; LNeu\%: lower in the percentage of neutrophil granulocytes; LNLR: lower in NLR.

\section{Declarations}

Ethics approval and consent to participate. The studies involving human participants were reviewed and approved by The Ethics Committee of Shandong Cancer Hospital Affiliated to Shandong First Medical University. The patients/participants provided agree to participate in this study.

\section{Consent for publication}

Not applicable.

\section{Availability of data and materials}

Al the data and material supporting the findings are present in the manuscript.

\section{Competing interests}

There is no conflict of interest among authors to disclose.

\section{Funding}

This project was support by the National Natural Science Foundation of China (81502667), Key Research and Development Plan of Shandong, China (2016GSF201167), and Natural Science Foundation of Shandong, China (2019PH111). The funding bodies had role in the study design and data collection.

\section{Authors' contributions}


Writing - original draft: XQX; Writing - review and editing: JMY, MHL and PX; Conceptualization: PX, XLL, WJT; Data collection and analysis: WJT, JLC; All authors have read and approved the final manuscript.

\section{Acknowledgements}

The authors would like to acknowledge Department of Radiation Oncology, Shandong Cancer Hospital \& Institute, for his organizational support.

\section{References}

1. Siegel, R.L., K.D. Miller, and A. Jemal, Cancer statistics, 2020. CA Cancer J Clin, 2020. 70(1): p. 7-30.

2. Berghmans, T., M. Paesmans, and J.P. Sculier, Prognostic factors in stage III non-small cell lung cancer: a review of conventional, metabolic and new biological variables. Ther Adv Med Oncol, 2011. 3(3): p. 127-38.

3. Sun, W., et al., Primary Tumor Location Is a Useful Predictor for Lymph Node Metastasis and Prognosis in Lung Adenocarcinoma. Clin Lung Cancer, 2017. 18(1): p. e49-e55.

4. Moon, Y., et al., Differing histopathology and prognosis in pulmonary adenocarcinoma at central and peripheral locations. J Thorac Dis, 2016. 8(1): p. 169-77.

5. Park, H.S., et al., Central versus Peripheral Tumor Location: Influence on Survival, Local Control, and Toxicity Following Stereotactic Body Radiotherapy for Primary Non-Small-Cell Lung Cancer. J Thorac Oncol, 2015. 10(5): p. 832-837.

6. Wang, Z., et al., Primary tumor location is an important predictor of survival in pulmonary adenocarcinoma. Cancer Manag Res, 2019. 11: p. 2269-2280.

7. Kim, J. and J.S. Bae, Tumor-Associated Macrophages and Neutrophils in Tumor Microenvironment. Mediators Inflamm, 2016. 2016: p. 6058147.

8. Helm, O., et al., Tumor-associated macrophages exhibit pro- and anti-inflammatory properties by which they impact on pancreatic tumorigenesis. Int J Cancer, 2014. 135(4): p. 843-61.

9. Noy, R. and J.W. Pollard, Tumor-associated macrophages: from mechanisms to therapy. Immunity, 2014. 41(1): p. 49-61.

10. Hanahan, D. and R.A. Weinberg, Hallmarks of cancer: the next generation. Cell, 2011. 144(5): p. 64674.

11. Prelaj, A., et al., EPSILoN: A Prognostic Score Using Clinical and Blood Biomarkers in Advanced NonSmall-cell Lung Cancer Treated With Immunotherapy. Clin Lung Cancer, 2020.

12. Castello, A., et al., The immune-metabolic-prognostic index and clinical outcomes in patients with non-small cell lung carcinoma under checkpoint inhibitors. J Cancer Res Clin Oncol, 2020. 146(5): p. 1235-1243.

13. Diem, S., et al., Neutrophil-to-Lymphocyte ratio (NLR) and Platelet-to-Lymphocyte ratio (PLR) as prognostic markers in patients with non-small cell lung cancer (NSCLC) treated with nivolumab. Lung Cancer, 2017. 111: p. 176-181. 
14. Mandaliya, $\mathrm{H}$., et al., Prognostic biomarkers in stage IV non-small cell lung cancer (NSCLC): neutrophil to lymphocyte ratio (NLR), lymphocyte to monocyte ratio ( $L M R)$, platelet to lymphocyte ratio (PLR) and advanced lung cancer inflammation index (ALI). Transl Lung Cancer Res, 2019. 8(6): p. 886-894.

15. Ono, T., et al., Impact of neutrophil-to-lymphocyte ratio in patients with EGFR-mutant NSCLC treated with tyrosine kinase inhibitors. Invest New Drugs, 2020. 38(3): p. 885-893.

16. Templeton, A.J., et al., Prognostic role of neutrophil-to-lymphocyte ratio in solid tumors: a systematic review and meta-analysis. J Natl Cancer Inst, 2014. 106(6): p. dju124.

17. Kiriu, T., et al., Pseudo-Progression and the Neutrophil-to-Lymphocyte Ratio in Non-Small Cell Lung Cancer Treated with Immune Checkpoint Inhibitors: A Case-Control Study. Onco Targets Ther, 2019. 12: p. 10559-10568.

18. Hasegawa, T., et al., Association of High Neutrophil-to-Lymphocyte Ratio With Poor Outcomes of Pembrolizumab Therapy in High-PD-L 1-expressing Non-small Cell Lung Cancer. Anticancer Res, 2019. 39(12): p. 6851-6857.

19. Xia, L.J., et al., Significance of neutrophil-to-lymphocyte ratio, platelet-to-lymphocyte ratio, lymphocyte-to-monocyte ratio and prognostic nutritional index for predicting clinical outcomes in T12 rectal cancer. BMC Cancer, 2020. 20(1): p. 208.

20. Lin, G.N., et al., Elevated neutrophil-to-lymphocyte ratio predicts poor outcome in patients with advanced non-small-cell lung cancer receiving first-line gefitinib or erlotinib treatment. Asia Pac J Clin Oncol, 2017. 13(5): p. e189-e194.

21. Meriggi, F., et al., Significance of neutrophil-to-lymphocyte ratio in Western advanced EGFR-mutated non-small cell lung cancer receiving a targeted therapy. Tumori, 2017. 103(5): p. 443-448.

22. Phan, T.T., et al., The prognostic impact of neutrophil to lymphocyte ratio in advanced non-small cell lung cancer patients treated with EGFR TKI. Int J Gen Med, 2018. 11: p. 423-430.

23. Aguiar-Bujanda, D., et al., Neutrophil to Lymphocyte Ratio as a Prognostic Factor in European Patients with Epidermal Growth Factor Receptor-Mutant Non-Small Cell Lung Cancer Treated with Tyrosine Kinase Inhibitors. Oncol Res Treat, 2018. 41(12): p. 755-761.

24. Deng, C., et al., High systemic immune-inflammation index predicts poor prognosis in advanced lung adenocarcinoma patients treated with EGFR-TKIs. Medicine (Baltimore), 2019. 98(33): p. e16875.

25. Nakamura, T., et al., The combined use of the neutrophil-lymphocyte ratio and C-reactive protein level as prognostic predictors in adult patients with soft tissue sarcoma. J Surg Oncol, 2013. 108(7): p. 481-5.

26. Morizawa, Y., et al., Correlation of Immune Cells and Cytokines in the Tumor Microenvironment with Elevated Neutrophil-To-Lymphocyte Ratio in Blood: An Analysis of Muscle-Invasive Bladder Cancer. Cancer Invest, 2018. 36(7): p. 395-405.

27. Tsai, M.S., et al., The prognosis of head and neck squamous cell carcinoma related to immunosuppressive tumor microenvironment regulated by IL-6 signaling. Oral Oncol, 2019. 91: p. 4755. 
28. Lee, K.H., et al., The prognostic and predictive value of tumor-infiltrating lymphocytes and hematologic parameters in patients with breast cancer. BMC Cancer, 2018. 18(1): p. 938.

29. Zhang, J., et al., Preoperative lymphocyte count is a favorable prognostic factor of disease-free survival in non-small-cell lung cancer. Med Oncol, 2013. 30(1): p. 352.

30. Cho, Y., et al., Impact of Treatment-Related Lymphopenia on Immunotherapy for Advanced Non-Small Cell Lung Cancer. Int J Radiat Oncol Biol Phys, 2019. 105(5): p. 1065-1073.

31. Iseki, Y., et al., The impact of the preoperative peripheral lymphocyte count and lymphocyte percentage in patients with colorectal cancer. Surg Today, 2017. 47(6): p. 743-754.

32. Jiang, H., et al., Association between EGFR exon 19 or exon 21 mutations and survival rates after first-line EGFR-TKI treatment in patients with non-small cell lung cancer. Mol Clin Oncol, 2019. 11(3): p. 301-308.

33. Zhang, Y., et al., Patients with exon 19 deletion were associated with longer progression-free survival compared to those with L858R mutation after first-line EGFR-TKIs for advanced non-small cell lung cancer: a meta-analysis. PLoS One, 2014. 9(9): p. e107161.

\section{Tables}


Table 1 Patients and clinical characteristics

\begin{tabular}{|c|c|}
\hline 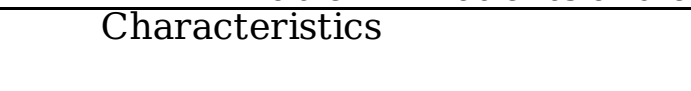 & $\begin{array}{l}\text { All patients }(\mathrm{n}=190) \\
\mathrm{n}(\%)\end{array}$ \\
\hline \multicolumn{2}{|l|}{ Gender } \\
\hline Male & $68(35.8 \%)$ \\
\hline Female & $122(64.2 \%)$ \\
\hline \multicolumn{2}{|l|}{ Age } \\
\hline Median & 58 \\
\hline Range & $34-87$ \\
\hline \multicolumn{2}{|l|}{ Smoking habits } \\
\hline Non-smoker & $148(77.9 \%)$ \\
\hline Ever-smoker & $42(22.1 \%)$ \\
\hline \multicolumn{2}{|l|}{ EGFR-mutation } \\
\hline L858R & $110(57.9 \%)$ \\
\hline 19del & $75(39.5 \%)$ \\
\hline Rare mutation & $5(2.6 \%)$ \\
\hline \multicolumn{2}{|l|}{ Stage } \\
\hline Postoperative recurrence & $26(13.7 \%)$ \\
\hline IV & $164(86.3 \%)$ \\
\hline \multicolumn{2}{|l|}{ EGFR Mutation Test } \\
\hline PCR & $172(90.5 \%)$ \\
\hline NGS & $18(9.5 \%)$ \\
\hline \multicolumn{2}{|l|}{ Tumor location } \\
\hline Central type & $48(25.3 \%)$ \\
\hline Peripheral type & $142(74.7 \%)$ \\
\hline \multicolumn{2}{|l|}{ Bone metastasis } \\
\hline YES & $83(43.7 \%)$ \\
\hline NO & $107(56.3 \%)$ \\
\hline \multicolumn{2}{|l|}{ Liver metastasis } \\
\hline YES & $26(13.7 \%)$ \\
\hline NO & $164(86.3 \%)$ \\
\hline \multicolumn{2}{|l|}{ Brain metastasis } \\
\hline YES & $59(31.1 \%)$ \\
\hline NO & $131(68.9 \%)$ \\
\hline \multicolumn{2}{|l|}{ First-line therapy } \\
\hline Surgery & $27(14.2 \%)$ \\
\hline Chemotherapy & $93(49.0 \%)$ \\
\hline Chemoradiotherapy & $9(4.7 \%)$ \\
\hline Radiotherapy combined targeted therapy & $15(7.9 \%)$ \\
\hline Targeted therapy & $36(18.9 \%)$ \\
\hline Radiotherapy & $6(3.2 \%)$ \\
\hline Chemotherapy combined with TKIs & $4(2.1 \%)$ \\
\hline \multicolumn{2}{|l|}{ Target therapy lines } \\
\hline First-line & $54(28.4 \%)$ \\
\hline Second-line & $102(53.7 \%)$ \\
\hline Third-line & $34(17.9 \%)$ \\
\hline
\end{tabular}

Table 2

Univariate and multivariate Cox proportional analysis regarding PFS $(n=190)$ 


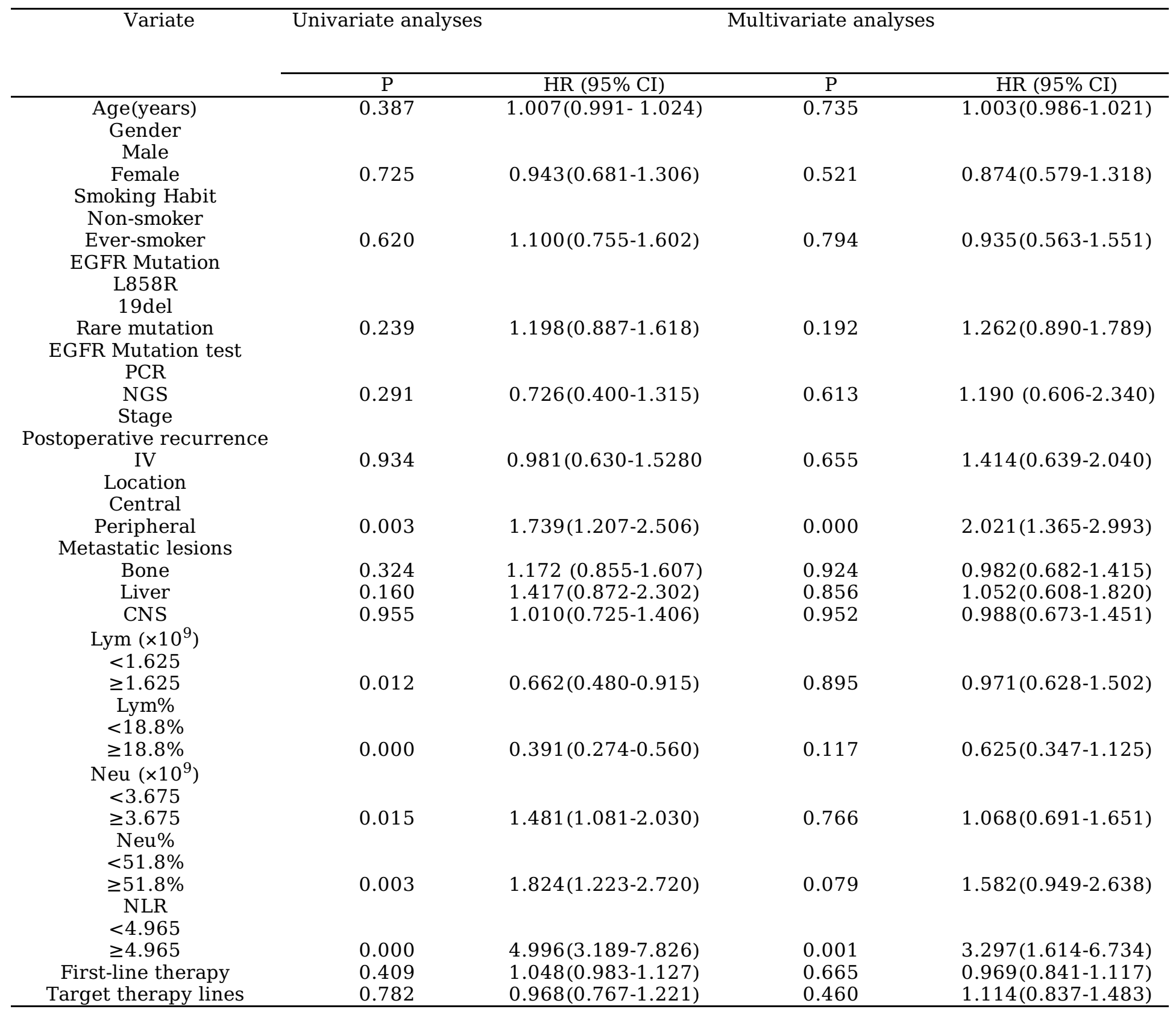

Table 3 The optimal cut-off values based on PFS

\begin{tabular}{llll}
\hline Peripheral blood index & Sensitivity & Specificity & AUC \\
\hline Lym $\left(\times 10^{9} / \mathrm{L}\right)$ & $63 \%$ & $64.4 \%$ & 0.632 \\
Lym\% & $88.9 \%$ & $28.2 \%$ & 0.549 \\
Neu $\left(\times 10^{9} / \mathrm{L}\right)$ & $59.3 \%$ & $54 \%$ & 0.521 \\
Neu\% & $88.9 \%$ & $19 \%$ & 0.499 \\
NLR & $17.8 \%$ & $100.0 \%$ & 0.550 \\
\hline
\end{tabular}

Table 4 Peripheral blood test with clinicopathological factors with $\mathrm{P}$ values 


\begin{tabular}{lllll}
\hline Parameter & R spearman & P value & U test & P value \\
\hline NLR\& Lym & -0.310 & 0.000 & & \\
NLR\& Neu & 0.595 & 0.000 & & \\
Lym\%\& Bone metastatic & & & 3617.500 & 0.029 \\
Neu\%\&Bone metastatic & & & 5442.5 & 0.008 \\
NLR\&Bone metastatic & & 5299.5 & 0.022 \\
\hline
\end{tabular}

\section{Figures}
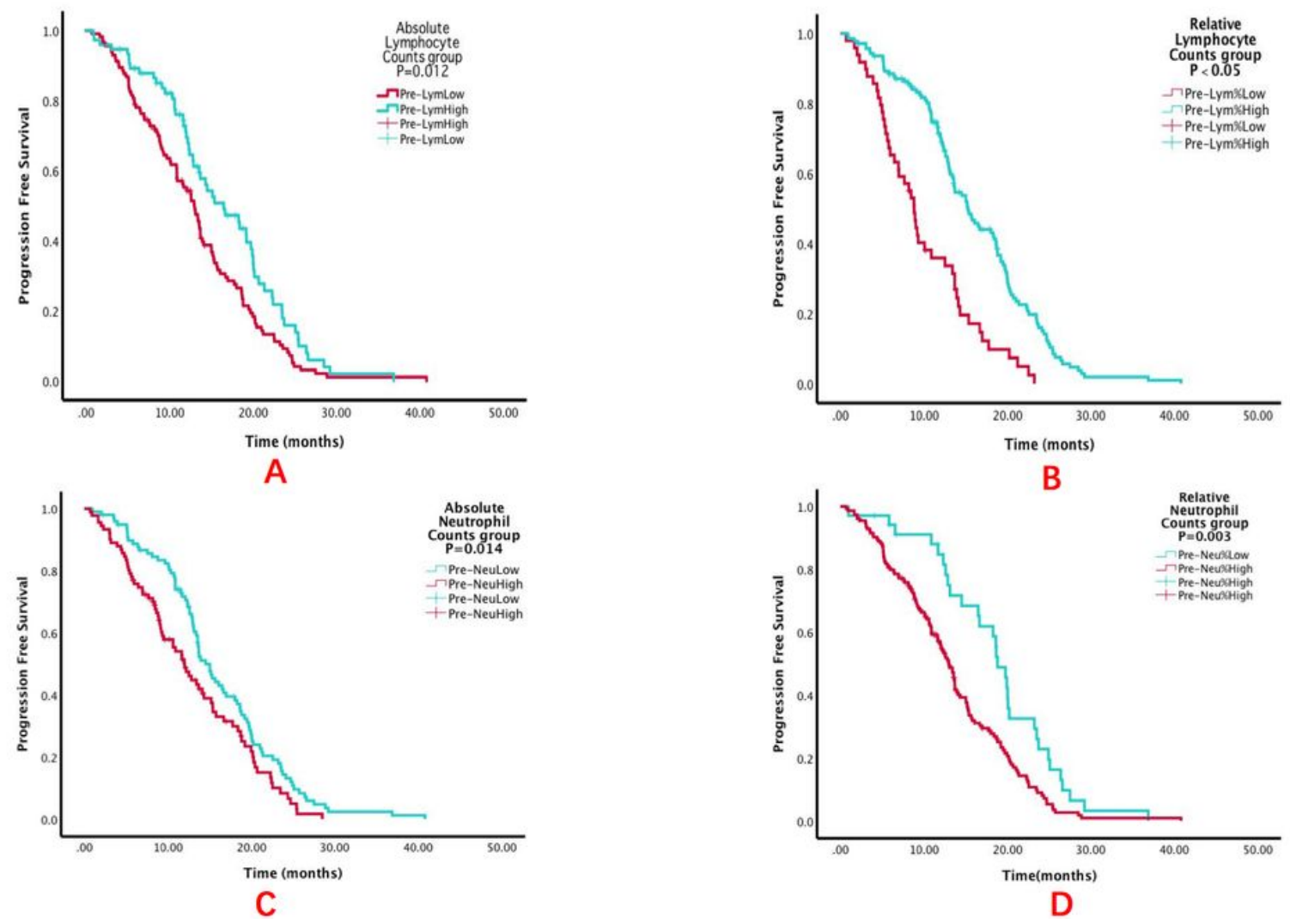

Figure 1

Kaplan-Meier plot of progression-free survival (PFS) stratified by pretreatment absolute lymphocyte counts group (A), relative lymphocyte counts group (B), absolute neutrophil counts group (C), and relative neutrophil counts group (D). 

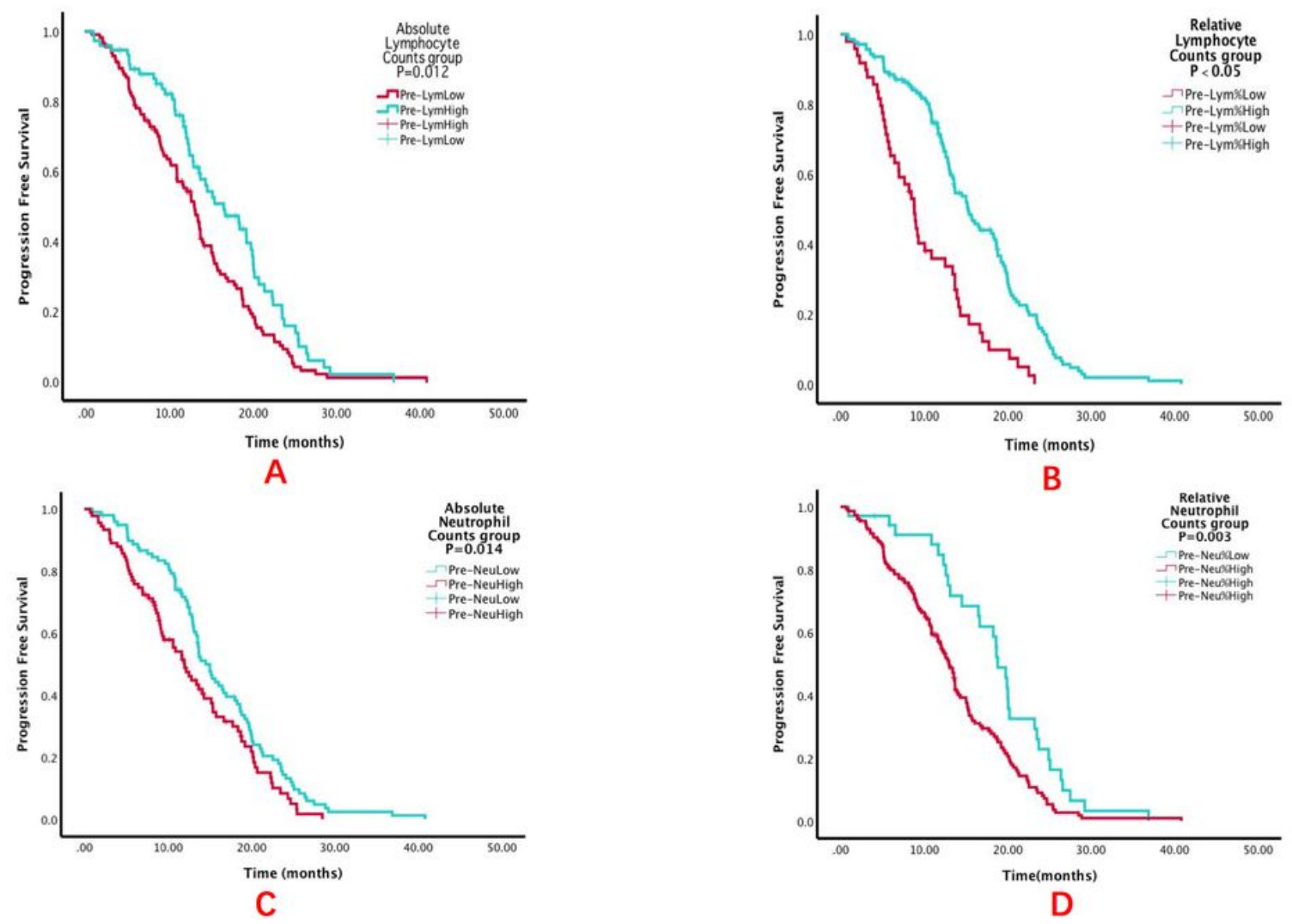

\section{Figure 1}

Kaplan-Meier plot of progression-free survival (PFS) stratified by pretreatment absolute lymphocyte counts group (A), relative lymphocyte counts group (B), absolute neutrophil counts group $(C)$, and relative neutrophil counts group (D). 


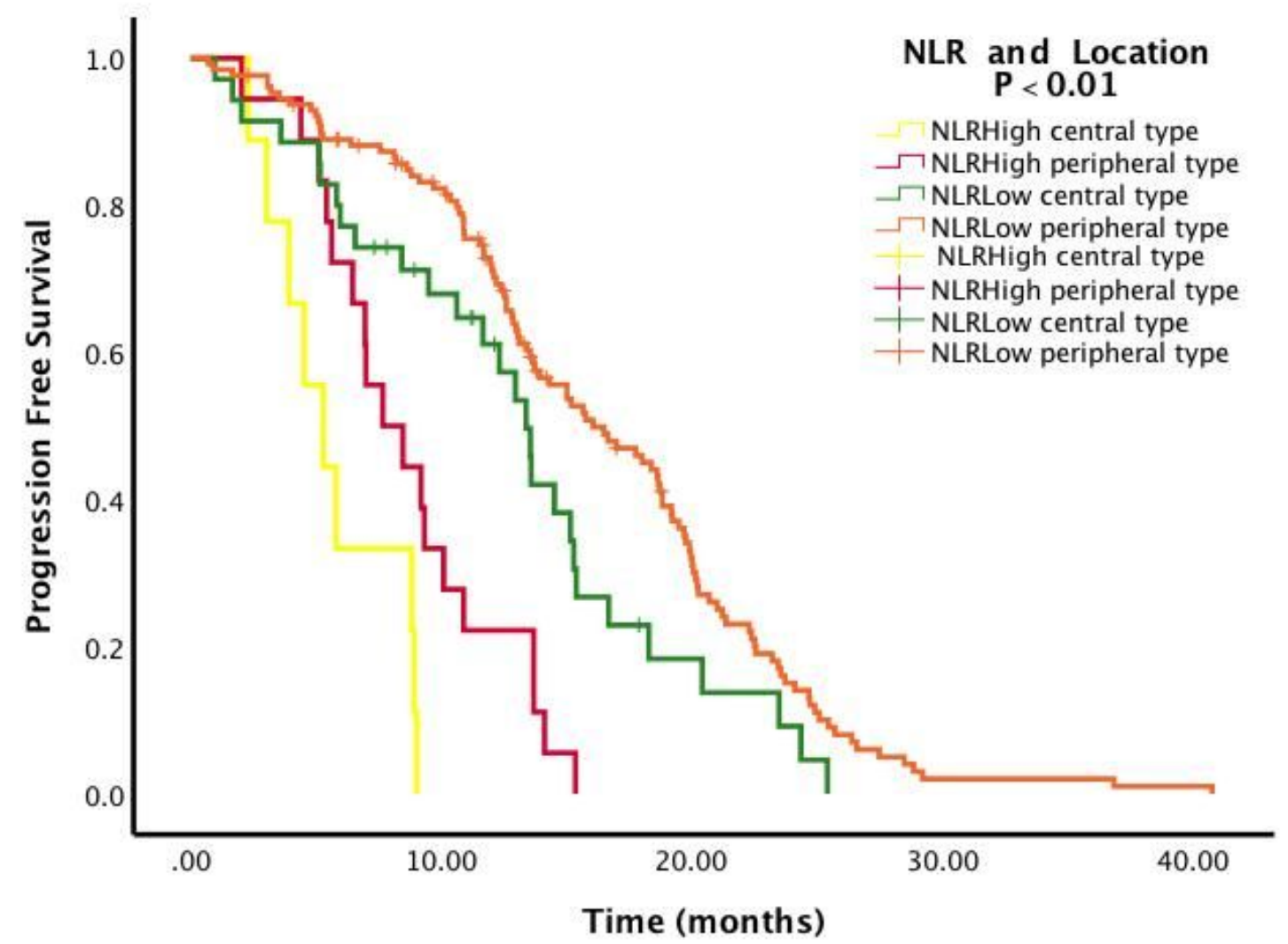

Figure 2

Kaplan-Meier survival curves of PFS based on combination of pretreatment neutrophil to lymphocyte expression and primary location in all patients. 


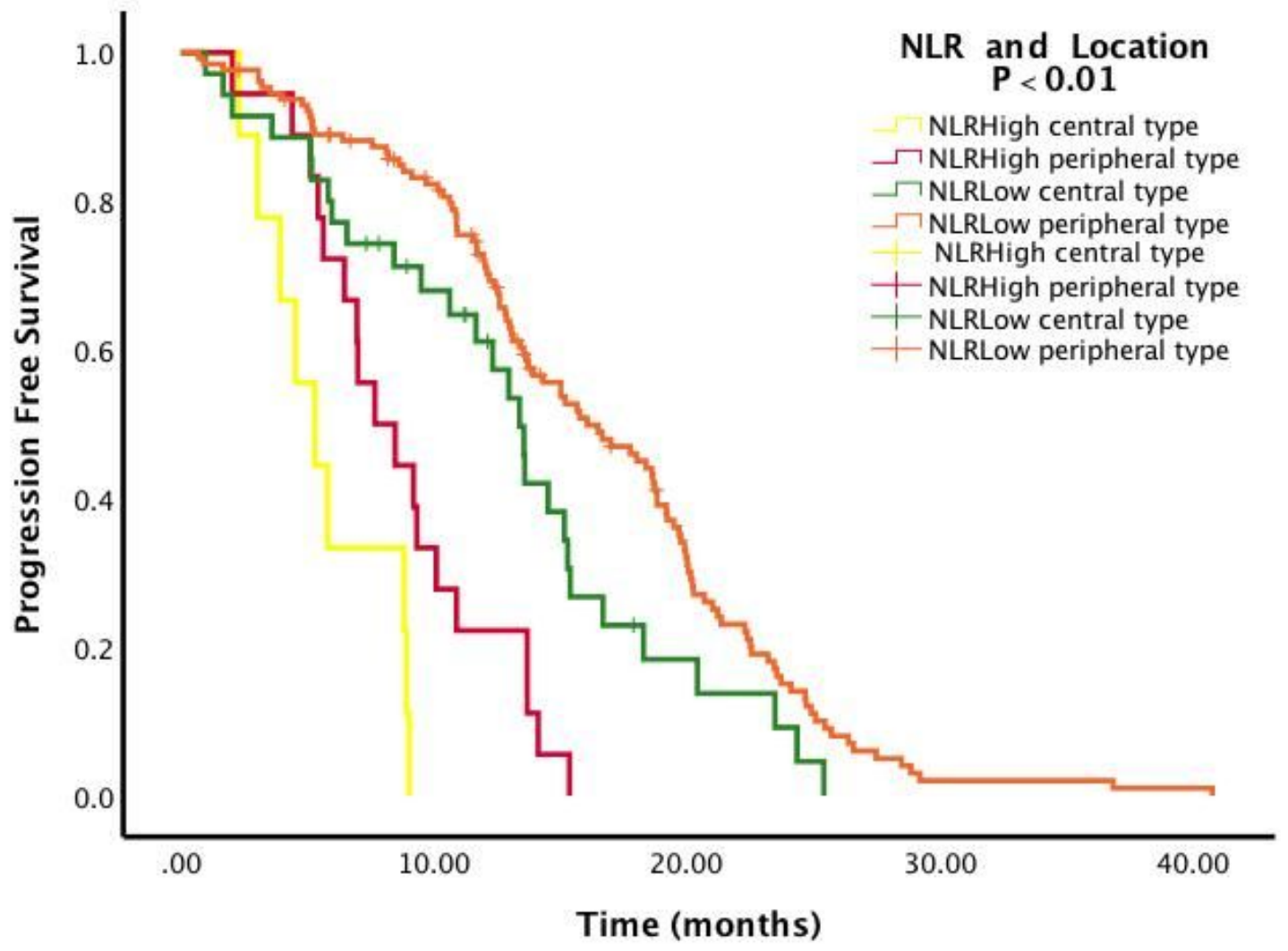

Figure 2

Kaplan-Meier survival curves of PFS based on combination of pretreatment neutrophil to lymphocyte expression and primary location in all patients.
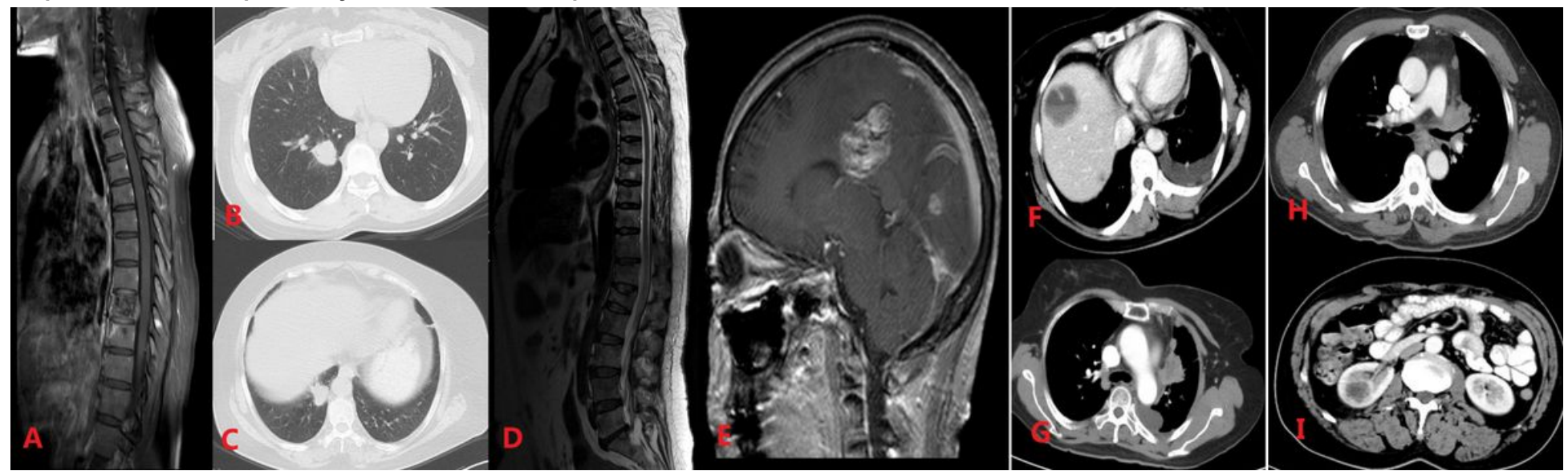

Figure 3

A patient with high NLR and peripheral type (B) in stage IV non-small cell lung cancer (A) with shorter PFS. A patient with low NLR and peripheral type (C) in stage IV lung adenocarcinoma with longer PFS. (D) A patient with low NLR and central (G) in stage IV lung adenocarcinoma (E, F) with shorter PFS. A patient with high NLR and central type $(\mathrm{H})$ in stage IV (I) with longer PFS. 

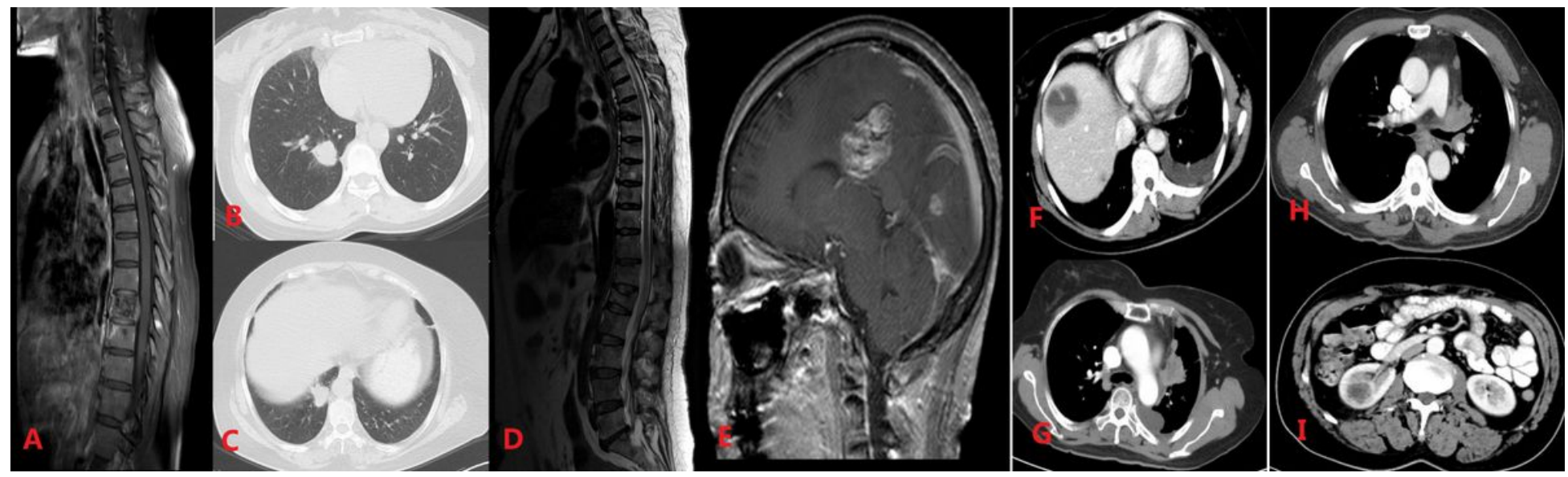

Figure 3

A patient with high NLR and peripheral type (B) in stage IV non-small cell lung cancer (A) with shorter PFS. A patient with low NLR and peripheral type (C) in stage IV lung adenocarcinoma with longer PFS. (D) A patient with low NLR and central (G) in stage IV lung adenocarcinoma (E, F) with shorter PFS. A patient with high NLR and central type $(\mathrm{H})$ in stage IV (I) with longer PFS. 